Article

\title{
Some New Integral Identities for Solenoidal Fields and Applications
}

\section{Vladimir I. Semenov}

Department of Applied Mathematics and Computer Sciences, Baltic Federal University, Alexander Nevskij st., 14, Kaliningrad 236041, Russia; E-Mail: visemenov @ rambler.ru

Received: 31 December 2013; in revised form: 7 February 2014 / Accepted: 19 February 2014/

Published: 3 March 2014

Abstract: In spaces $R^{n}, n \geq 2$, it has been proved that a solenoidal vector field and its rotor satisfy the series of new integral identities which have covariant form. The interest in them is explained by hydrodynamics problems for an ideal fluid.

Keywords: rotor; solenoidal vector field; potential vector field; Euler equations; Navier-Stokes equations

\section{Introduction}

In [1] , Dobrokhotov and A. Shafarevich found the interesting property of solutions in the Cauchy problem for the Navier-Stokes equations in space. If $u=\left(u_{1}, u_{2}, u_{3}\right)$ is a fluid velocity then

$$
\int_{R^{3}} u_{i} u_{k} d x=\frac{\delta_{k i}}{3} \int_{R^{3}}|u|^{2} d x
$$

where $\delta_{k i}$ is the Kronecker symbol. In other words, we have a conservation of some things close to conformal properties. Later, in [2], L. Brandolese confirmed this result for dimensions $n \geq 4$ (see also [3]). In fact, this is the property of solenoidal vector fields if a potential part of a mapping

$$
u_{i} \frac{\partial u}{\partial x_{i}}:=u_{i} u_{, i}
$$

is summaable to a power $r>1$ (see [4] ). Here, the repeated index means summation.

Now, I would like to consider other things connected with integral identities. A part of them was studied by author in [4]. Every finite smooth solenoidal vector field $u$ satisfies the following integral identity:

$$
\int_{R^{n}} u_{i, j} u_{k, i} u_{k, j} d x=-\int_{R^{n}} u_{i} u_{k, i} \triangle u_{k} d x
$$


(see [4] where some statements connected with this formula are given). This very simple formula implies some new identities and applications to the 2d Navier-Stokes and Euler equations. In particular, we obtain exact a priori estimates in Ladyzchenskaya's and Judovich's theorems.

\subsection{Notations}

Let $u: R^{n} \rightarrow R^{n}, u=\left(u_{1}, u_{2}, \cdots, u_{n}\right), n \geq 2$, be an arbitrary vector field. Symbols

$$
u_{k, i}=\frac{\partial u_{k}}{\partial x_{i}}, u_{k, i j}=\frac{\partial^{2} u_{k}}{\partial x_{i} \partial x_{j}}
$$

and so forth mean a partial differentiation or differentiation in distributions. Naturally, $\Delta$ is the Laplace operator. Below, unless otherwise indicated, the repeated indices mean summation. For example,

$$
u_{i} u_{j, i}=\sum_{i=1}^{n} u_{i} u_{j, i}, u_{i, j} u_{j, i}=\sum_{i, j=1}^{n} u_{i, j} u_{j, i}, u_{i} u_{j, i} \triangle u_{j}=\sum_{i, j=1}^{n} u_{i} u_{j, i} \triangle u_{j}
$$

etc. Further, I consider rotor coordinates (for dimension $n=2,3$ )

$$
c_{k i}(u)=u_{k, i}-u_{i, k}
$$

as elements of a skew-symmetric matrix $C$. The Jacobi matrix in distributions of the vector field $u$ is denoted by $\nabla u$. As in common practice, the modulus of a matrix $A$ is defined by the equality

$$
|A|=\left(\sum_{i, j} a_{i j}^{2}\right)^{\frac{1}{2}}
$$

A symbol $W_{p}^{l}\left(R^{n}\right)$ denotes the Sobolev class of vector fields which have all derivatives in distributions until an order $l$ and summable to a power $p \geq 1$. The norm in this space is given by formula:

$$
\|v\|_{W_{p}^{l}\left(R^{n}\right)}=\sum_{|\alpha| \leq l}\left\|D^{\alpha} v\right\|_{p}
$$

where a symbol $\|h\|_{p}$ is a norm in space $L_{p}\left(R^{n}\right)$.

Respectively, a class of infinitely smooth vector fields with a compact support is denoted by $C_{0}^{\infty}\left(R^{n}\right)$ and a closure of this set in the norm of the space $W_{p}^{l}\left(R^{n}\right)$ is written by $\stackrel{\circ}{W_{p}^{l}}\left(R^{n}\right)$.

\section{Solenoidal Vector Fields and Integral Identities for Dimension $n \geq 2$}

A classical integral identity for these fields goes on the Helmholtz-Weyl theorem about the decomposition of a smooth vector field by the sum of potential and solenoidal terms. Later, (see [5,6], p. 339) it was shown that the space $L_{2}\left(R^{3}\right)$ of vector fields has decomposition $L_{2}\left(R^{3}\right)=J \oplus G$ where subspaces $J$ and $G$ are closures in $L_{2}\left(R^{3}\right)$ of finite, smooth solenoidal and potential vector fields respectively.

The main results are described by theorems 1 and 2 . 
Theorem 1. Suppose, that solenoidal vector field $u \in \stackrel{\circ}{W_{2}^{3}}\left(R^{n}\right), n \geq 2$. Then integral identities are true:

$$
\begin{gathered}
\int_{R^{n}} \Delta u_{i, j} c_{k i}(u) c_{k j}(u) d x=0 \\
\int_{R^{n}}\left(c_{i m, j}(u)+c_{j m, i}(u)\right) c_{k i}(u) c_{k j, m}(u) d x=0 \\
\int_{R^{n}} u_{i, j m} u_{k, i m} u_{k, j} d x=0 \\
\int_{R^{n}}\left(\Delta u_{i, j} u_{k, i} u_{k, j}+u_{i, j} \Delta u_{k, i} u_{k, j}\right) d x=\int_{R^{n}} u_{i, j} u_{k, i} \Delta u_{k, j} d x \\
\int_{R^{n}} u_{i, j} c_{k i}(\Delta u) c_{k j}(u) d x=\int_{R^{n}} u_{i, j} c_{k i}(u) c_{k j}(\Delta u) d x
\end{gathered}
$$

If $u \in \stackrel{\circ}{W_{2}^{4}}\left(R^{n}\right), n \geq 2$, then

$$
\int_{R^{n}} u_{i} u_{k, i} \Delta^{2} u_{k} d x=-\int_{R^{n}}\left(u_{i, j} \Delta u_{k, i} u_{k, j}+u_{i, j} u_{k, i} \Delta u_{k, j}\right) d x
$$

The proof of this theorem relies on the following statement.

Lemma 1. If a vector field $u \in W_{p}^{2}\left(R^{n}\right), n \geq 2, p \geq 1$, then vector field $g=\left(g_{1}, g_{2}, \cdots, g_{n}\right)$ where $g_{i}=c_{k i, j} c_{k j}|C|^{p-2}, c_{k j}$ from formula (1), the matrix $C$ is defined above, is potential i.e., for every smooth solenoidal vector field $v \in C_{0}^{\infty}\left(R^{n}\right)$ the integral identity is fulfilled:

$$
\int_{R^{n}} v_{i} c_{k i, j}(u) c_{k j}(u)|C|^{p-2} d x=0
$$

Proof of Lemma 1. It is sufficient to see the equality:

$$
c_{k i, j} c_{k j}=\frac{1}{4} \sum_{k, j=1}^{n} \frac{\partial}{\partial x_{i}}\left(u_{k, j}-u_{j, k}\right)^{2}
$$

which follows from relations of the type: $u_{i, k j} u_{j, k}=u_{i, j k} u_{k, j}$.

Proof of Theorem 1. Without any restrictions we assume that a vector field $u \in C_{0}^{\infty}\left(R^{n}\right)$ (see [7], Theorem 1). In the equality of lemma 1 we take: $p=2, v=\Delta u$. Further, we integrate by parts with respect to variable $x_{j}$. Since $c_{k j, j}(u)=\Delta u_{k}$ and $c_{k i, j j}(u)=c_{k i}(\Delta u)$ then we obtain equation (3). Applying $\Delta u_{i, j}=c_{i m, m j}(u)$ for substitution to equation (3) and integrating by parts with respect to variable $x_{m}$ we exchange summation indices $i$ and $j$ in the first product. Then it follows equation (4).

For the proof of equation (5) we note sums equality: $u_{i, j m} u_{k, i m} u_{k, j}=u_{i, m j} u_{k, i j} u_{k, m}$. Then

$$
2 \int_{R^{n}} u_{i, j m} u_{k, i m} u_{k, j} d x=\int_{R^{n}} u_{i, j m}\left(u_{k, m} u_{k, j}\right)_{, i} d x=0
$$

since div $u_{, j m}=0$.

Now, we write equation (3) by expanded form:

$$
\int_{R^{n}}\left(\Delta u_{i, j} u_{k, i} u_{k, j}-\Delta u_{i, j} u_{k, i} u_{j, k}-\Delta u_{i, j} u_{i, k} u_{k, j}+\Delta u_{i, j} u_{i, k} u_{j, k}\right) d x=0
$$


In the third term we exchange summation indices $i$ and $k$. In the forth term we make it twice. By the first step we exchange $i$ and $k$, after that $i$ and $j$. The second term vanishes because

$$
\begin{gathered}
\int_{R^{n}} \Delta u_{i, j} u_{k, i} u_{j, k} d x=-\int_{R^{n}} \Delta u_{i} u_{k, i j} u_{j, k} d x= \\
=-\frac{1}{2} \int_{R^{n}} \Delta u_{i} \frac{\partial}{\partial x_{i}}\left(u_{k, j} u_{j, k}\right) d x=0
\end{gathered}
$$

Therefore, the previous equality reduces to formula (6).

For verification (8) we use identity:

$$
\int_{R^{n}} u_{i} u_{k, j m i} u_{k, j m} d x=0
$$

where we integrate by parts. Then

$$
\int_{R^{n}} u_{i, m} u_{k, j i} u_{k, j m} d x+\int_{R^{n}} u_{i} u_{k, j i} \Delta u_{k, j} d x=0
$$

Here, for every integral we apply the integration by parts with respect to variable $x_{j}$. As the result we get:

$$
\begin{aligned}
\int_{R^{n}} u_{i} u_{k, i} \Delta^{2} u_{k} d x= & -\int_{R^{n}} u_{i, j} \Delta u_{k, i} u_{k, j} d x-\int_{R^{n}} u_{i, m j} u_{k, i m} u_{k, j} d x- \\
& -\int_{R^{n}} u_{i, m} u_{k, i} \Delta u_{k, m} d x
\end{aligned}
$$

On the right hand side, the middle integral vanishes by formula (5). In the third integral in the same place we exchange index $m$ by index $j$. Hence, we have equation (8).

Corollary 1. Let $u \in \stackrel{\circ}{W_{2}^{6}}\left(R^{n}\right), n \geq 2$, be a solenoidal vector field. Then the integral identity is true:

$$
\int_{R^{n}}\left(u_{k, i} \Delta^{2} u_{i, j}+2 \Delta u_{i, j} \Delta u_{k, i}+u_{i, j} \Delta^{2} u_{k, i}\right) u_{k, j} d x=\int_{R^{n}} u_{i, j} u_{k, i} \Delta^{2} u_{k, j} d x
$$

Proof of Corollary 1. Let $T=T(t, x)$ be a solution of equation $T=\nu \Delta T$ with an initial data $T(0, x)=u(x)$. Now, we rewrite equation (6) for the solenoidal vector field $T$ and differentiate it with respect to $t$. A passage to the limit as $t \rightarrow 0$ gives the necessary equality.

\section{Solenoidal Vector Fields and Integral Identities for Dimension $n=2$}

In this case we can give more precise identities. Applying them we can obtain the exact estimates for solutions in the Cauchy problem for the Navier-Stokes equations and Euler equations.

Theorem 2. Let $u, v$ be solenoidal vector fields in $R^{2}$.

(1) If $u, v \in W_{2}^{1}\left(R^{2}\right)$ then almost everywhere there are fulfilled:

$$
u_{i, j} u_{k, i} v_{k, j}=0, v_{i, j} c_{k i}(u) c_{k j}(u)=0
$$

(2) If $u, v \in \stackrel{\circ}{W_{2}^{3}}\left(R^{2}\right)$ then the following integral identities are true:

$$
\int_{R^{2}} v_{i} c_{k i}(u) \Delta u_{k} d x=0
$$




$$
\int_{R^{2}}\left(\Delta u_{i, j} u_{k, i} u_{k, j}+u_{i, j} \Delta u_{k, i} u_{k, j}\right) d x=0
$$

(3) If $u \in \stackrel{\circ}{W_{2}^{5}}\left(R^{2}\right)$ then

$$
\begin{gathered}
\int_{R^{2}}\left(\Delta^{2} u_{i, j} u_{k, i} u_{k, j}+u_{i, j} \Delta^{2} u_{k, i} u_{k, j}\right) d x=0 \\
\int_{R^{2}} u_{i} u_{k, i} \Delta^{2} u_{k} d x=-\int_{R^{2}} u_{i, j} \Delta u_{k, i} u_{k, j} d x=\int_{R^{2}} \Delta u_{i, j} u_{k, i} u_{k, j} d x
\end{gathered}
$$

Proof of Theorem 2. Direct calculations and equalities div $u=0$, div $v=0$ prove equation (9). Since

$$
\int_{R^{2}} v_{i, j} c_{k i}(u) c_{k j}(u) d x=0
$$

then integrating by parts we get equation (10). Formula (11) follows from equations (9) and (6) where $v=\Delta u$. Identity (12) we obtain from corollary 1 and equation (9) where we must replace $u$ by $\Delta u, v$ by $u$. Finally, equation (13) we have from equation (8) and the first identity from equation (9).

\section{Applications}

Let us consider the Cauchy problem for the Navier-Stokes equations

$$
\begin{gathered}
\dot{u}+u_{i} u_{, i}=\nu \Delta u-\nabla p \\
d i v u=0, u(0, x)=\varphi(x)
\end{gathered}
$$

if dimension $n=2$ or $n=3$. We also suppose that an initial data $\varphi \in C_{0}^{\infty}\left(R^{n}\right)$. An existence of a weak solutions for small time interval was proved in [8] (their regularity it was shown in [9]). There are some conditions of an existence of global regular solutions.

Now, we note only monotonicity properties of regular solutions. Every regular solution satisfies the conditions:

$$
\begin{gathered}
\frac{1}{2} \frac{d}{d t}\|\nabla u\|_{2}^{2}=\int_{R^{2}} u_{i} u_{k, i} \Delta u_{k} d x-\nu\|\Delta u\|_{2}^{2} \\
\frac{1}{2} \frac{d}{d t}\|\Delta u\|_{2}^{2}=-\int_{R^{3}} u_{i} u_{k, i} \Delta^{2} u_{k} d x-\nu\|\nabla \Delta u\|_{2}^{2}
\end{gathered}
$$

Integrals finiteness in these formulas follows from [10].

Theorem 3. Let be an initial data $\varphi \in C_{0}^{\infty}\left(R^{n}\right)$. Suppose, that u is a regular solution of the problem (14) and (15). If $n=2$ then a function

$$
\eta(t)=\|\nabla u\|_{2}^{2}
$$

is a decreasing function.

If $n=3$ and

then a function

$$
\|\varphi\|_{2}^{3}\|\Delta \varphi\|_{2} \leq \frac{4 \sqrt{3}}{3} \nu^{4}
$$

$$
\omega(t)=\|u\|_{2}^{3}\|\Delta u\|_{2}
$$

is a decreasing function. 
Proof. We take $v=u$ in formula (10) then from (16) it can be deduced the inequality

$$
\frac{1}{2} \frac{d}{d t}\|\nabla u\|_{2}^{2}=-\nu\|\Delta u\|_{2}^{2}
$$

Hence, we have the first statement.

To prove the second part we combine (16) and (13). Then

$$
\int_{R^{3}} u_{i} u_{k, i} \Delta^{2} u_{k} d x=-2 \int_{R^{3}} u_{i, j} u_{k, i m} u_{k, j m} d x
$$

Further, we apply Hölder's inequality. Therefore,

$$
\begin{gathered}
\frac{1}{2} \frac{d}{d t}\|\Delta u\|_{2}^{2} \leq 2 \int_{R^{3}}\left|\nabla u\left\|\left.\nabla(\nabla u)\right|^{2} d x-\nu\right\| \nabla \Delta u \|_{2}^{2} \leq\right. \\
\leq 2\|\nabla u\|_{2}\|\nabla(\nabla u)\|_{4}^{2}-\nu\|\nabla \Delta u\|_{2}^{2}
\end{gathered}
$$

Use the estimate from [9] (see p. 74). Then

$$
\|v\|_{4}^{2} \leq \sqrt{2} \cdot 3^{-3 / 4}\|v\|_{2}^{1 / 2}\|\nabla v\|_{2}^{3 / 2}
$$

where $v=\nabla(\nabla u)$. (For $n=3$ the factor 2 is omitted among intermediate calculations there.) Since $\|\nabla \nabla u\|_{2}=\|\Delta u\|_{2},\|\nabla(\nabla(\nabla u))\|_{2}=\|\nabla \Delta u\|_{2}$, then finding a function maximum $f(z)=b z^{3 / 2}-\nu z^{2}$, where $z=\|\nabla \Delta u\|_{2}$, we get:

$$
\frac{1}{2} \frac{d}{d t}\|\Delta u\|_{2}^{2} \leq \frac{3 \sqrt{3}}{4}\|\nabla u\|_{2}^{4}\|\Delta u\|_{2}^{2} \leq \frac{3 \sqrt{3}}{4}\|u\|_{2}\|\nabla u\|_{2}^{2}\|\Delta u\|_{2}^{3}
$$

Then for the function $\omega(t)=\|u\|_{2}^{3}\|\Delta u\|_{2}$ we have:

$$
\frac{d \omega}{d t} \leq 3\|u\|_{2}\|\nabla u\|_{2}\|\Delta u\|_{2}\left(-\nu+\frac{\sqrt{3}}{4 \nu} \omega(t)\right)
$$

The inequality follows from a nonpositiveness of the derivative $\omega^{\prime}$.

Remark 1. Monotonicity properties are very important for the exact a priory estimates for solutions of Navier-Stokes equations. For example, if dimension $n=2$, we get

$$
\|\nabla u\|_{2}^{2} \leq\|\nabla \varphi\|_{2}^{2}
$$

in the problem (14) and (15).

Remark 2. Obvious and exact estimates may be obtained for weak solutions of Euler equations (see [4]) and Navier-Stokes equations with an outer force.

Remark 3. If dimension $n=3$ then from Gagliardo's and Nirenberg's inequalities (see [11] and [12]) with some constant $C$ we get for solution in the problem (14) and (15) the following uniform estimate

$$
\|\nabla u\|_{2}^{2} \leq C\|\varphi\|_{2}\|\Delta \varphi\|_{2}
$$


Theorem 4. Let be dimension $n=2$ and a solenoidal vector field $u \in \stackrel{\circ}{W_{2}^{2}}\left(R^{2}\right)$. Then a vector field

$$
w=\Delta u_{i} u_{, i}+u_{i, j} u_{, i j}
$$

is a potential field in distributions.

Proof. It follows from the first equality (9) where a solenoidal vector field $v \in C_{0}^{\infty}\left(R^{2}\right)$. Since

$$
\int_{R^{2}} u_{i, j} u_{k, i} v_{k, j} d x=0
$$

then

$$
\int_{R^{2}} w_{k} v_{k} d x=0
$$

Therefore, the statement is proved.

\section{Conflicts of Interest}

The author declares no conflict of interest.

\section{References}

1. Dobrokhotov, S.J.; Shafarevich, A.I. On the behavior of an incompressible fluid velocity field at infinity. Fluid Dyn. 1996, 4, 511-514.

2. Brandolese, L. On the localization of symmetric and asymmetric solutions of the Navier-Stokes equations in $R^{n}$. Comptes Rendus Acad. Sci. Ser. I 2001, 332, 125-130.

3. Pukhnachev, V.V. Integral of motions of ideal liquid in the whole space. J. Appl. Mech. Tech. Phys. 2004, 2, 22-27, (in Russian).

4. Semenov, V.I. Some general properties of solenoidal vector fields and their applications to the $2 \mathrm{~d}$ Navier-Stokes and Euler equations. Nauchnye Vedom. Belgorodskogo Gos. Univ. Ser. Mat. 2008, 13, 109-129, (in Russian).

5. Sobolev, S.L. One new problem of mathematical physics. Izv. Akad. Nauk SSSR Ser. Mat. 1954, 18, 3-50, (in Russian).

6. Sobolev S.L. One new problem of mathematical physics. In: Izbrannye Trudy, 1 (in Russian); Sobolev Institute of Mathematics: Novosibirsk, Russia, 2003; pp. 32-58.

7. Maslennikova, V.N.; Bogovskij, M.E. Approximation of potential and solenoidal vector fields. Sib. Math. J. 1983, 24, 768-787.

8. Kiselev, A.A.; Ladyzhenskaya, O.A. On existence and uniqueness of solution in nonstationary problem for viscous incompressible fluid. Izv. Akad. Nauk SSSR Ser. Mat. 1957, 21, 655-680, (in Russian).

9. Serrin, J. The initial value problem for the Navier-Stokes equations. In Nonlinear Problems; Langer, R., Ed.; University of Wisconsin Press: Madison, WI, USA, 1963; pp. 69-98.

10. Fabes, E.; Jones, B.; Riviere, N. The initial value problem for the Navier-Stokes equations with data in $R^{n}$. Arch. Ration. Mech. Anal. 1972, 45, 222-240. 
11. Gagliardo, E. Ulterori proprietà di alcune classi di funzioni in più variabiliti. Ricerche di Mat. 1959, 45, 24-51.

12. Nirenberg, L. On elliptic partial differential equations. Ann. Scuola. Norm. Sup. di Pisa Ser. III,13, Fasc. II 1959, 115-162.

(C) 2014 by the author; licensee MDPI, Basel, Switzerland. This article is an open access article distributed under the terms and conditions of the Creative Commons Attribution license (http://creativecommons.org/licenses/by/3.0/). 